Bulletin of the Section of Logic

Volume 50/4 (2021), pp. 397-419

https://doi.org/10.18778/0138-0680.2021.01

Narges Akhlaghinia

Mona Aaly Kologani (D)

Rajab Ali Borzooei

Xiao Long Xin

\title{
ON THE CATEGORY OF EQ-ALGEBRAS
}

\begin{abstract}
In this paper, we studied the category of $E Q$-algebras and showed that it is complete, but it is not cocomplete, in general. We proved that multiplicatively relative $E Q$-algebras have coequlizers and we calculated coproduct and pushout in a special case. Also, we constructed a free $E Q$-algebra on a singleton.

Keywords: $E Q$-algebras, free $E Q$-algebras, category theory, universal algebra, variety.

2010 Mathematical Subject Classification: 06E15, 06F99.

\section{Introduction}

Fuzzy type theory was developed as a counterpart of the classical higherorder logic. Since the algebra of truth values is no longer a residuated lattice, a specific algebra called an $E Q$-algebra was proposed by Novák $[16,17,18]$. The main primitive operations of $E Q$-algebras are meet, multiplication, and fuzzy equality. Implication is derived from fuzzy equality and it is not a residuation with respect to multiplication. Consequently, $E Q$-algebras overlap with residuated lattices but are not identical to them. Novák and De Baets in [18] introduced various kinds of EQ-algebras.
\end{abstract}

Presented by: Jie Fang

Received: June 13, 2020

Published online: January 20, 2021

(C) Copyright by Author(s), Łódź 2021

(C) Copyright for this edition by Uniwersytet Łódzki, Łódź 2021 
Novák and El-Zekey in [14], proved that the class of $E Q$-algebras is a variety. In [19], J. Yang and X. Zhang introduced a new class of $E Q$-algebras, i.e., multiplicatively relative $E Q$-algebras. Also, they defined the notion of a filter generated by a nonempty subset.

Category theory is a powerful language, or conceptual framework, allowing us to see the universal components of a family of structures of a given kind, and how structures of different kinds are interrelated. Category theory is both an interesting object of philosophical study, and a potentially powerful formal tool for philosophical investigations of concepts such as space, system, and even truth. In [1], it has shown that the variety algebras, together with its homomorphisms, form a category and also, every non-trivial variety of algebras contains a free object on a given set. The category of some algebraic structures are studied. It is well known that the category of groups, rings, modules, and vector spaces are complete and cocomplete. The category of some logical algebraic structures have been studied well, too. For example, it has been proved that the category of Boolean algebras is isomorphic to the subcategory of rings named as Boolean rings [8]. Also, it is known that the category of $M V$-algebras is equivalent to that of unital lattice ordered groups ( $\ell$-groups). This equivalence, which depends in large part on the natural algebraic addition of $M V$-algebras [9] has been an essential tool in the study of $M V$-algebras. The categories of some other algebraic structures such as BCK(BCI)-algebras, BL-algebras, soft and rough sets have been studied, too (see $[5,6,7,10,11,12]$ ).

With these inspirations, we studied the category of $E Q$-algebras and showed that it is complete, but it is not cocomplete, in general. We proved that multiplicatively relative $E Q$-algebras have coequlizers and we calculated coproduct and pushout in a special case. Also, we constructed a free $E Q$-algebra on a singleton.

\section{Preliminaries}

In this section, we recollect some definitions and results which will be used in this paper (See $[13,14,19]$ ).

An $E Q$-algebra is an algebraic structure $\mathcal{E}=(E, \wedge, \otimes, \sim, 1)$ of type $(2,2,2,0)$, where for any $a, b, c, d \in E$, the following statements hold:

$(E 1)(E, \wedge, 1)$ is a $\wedge$-semilattice with top element 1 . For any $a, b \in E$, we set $a \leqslant b$ if and only if $a \wedge b=a$. 
(E2) $(E, \otimes, 1)$ is a (commutative) monoid and $\otimes$ is isotone with respect to $\leqslant$.

(E3) $a \sim a=1$.

$(E 4)((a \wedge b) \sim c) \otimes(d \sim a) \leqslant(c \sim(d \wedge b))$.

$(E 5)(a \sim b) \otimes(c \sim d) \leqslant(a \sim c) \sim(b \sim d)$.

(E6) $(a \wedge b \wedge c) \sim a \leqslant(a \wedge b) \sim a$.

(E7) $a \otimes b \leqslant a \sim b$.

The operations " $\wedge ", " \otimes "$, and " $\sim$ "are called meet, multiplication, and fuzzy equality, respectively. For any $a, b \in E$, we defined the binary operation implication on $E$ by, $a \rightarrow b=(a \wedge b) \sim a$. Also, in particular $1 \rightarrow a=1 \sim a=\tilde{a}$.

Let $\mathcal{E}=(E, \wedge, \otimes, \sim, 1)$ be an $E Q$-algebra and $a, b, c, d \in E$ are arbitrary elements. Then $\mathcal{E}$ is called separated, if $a \sim b=1$, then $a=b$, good, if $\tilde{a}=a$, residuated, where $(a \otimes b) \wedge c=a \otimes b$ if and only if $a \wedge((b \wedge c) \sim b)=a$, lattice-ordered $E Q$-algebra, if it has a lattice reduct, ${ }^{1}$, lattice $E Q$-algebra (or $\ell E Q$-algebra). if it is a lattice-ordered $E Q$-algebra and

$$
((a \vee b) \sim c) \otimes(d \sim a) \leqslant((d \vee b) \sim c),
$$

multiplicatively relative EQ-algebra, if $a \sim b \leqslant(a \otimes c) \sim(b \otimes c)$.

Proposition 2.1 ([14]). Let $\mathcal{E}$ be an $E Q$-algebra. Then for any $a, b, c \in E$, $\mathcal{E}$ is residuated if and only if $\mathcal{E}$ is good and $(a \otimes b) \rightarrow c \leqslant a \rightarrow(b \rightarrow c)$.

Proposition 2.2 ([19]). Each linear and residuated EQ-algebra is multiplicatively relative.

Proposition 2.3 ([14]). Let $\mathcal{E}$ be an $E Q$-algebra. Then, for all $a, b, c \in E$ (i) $a \sim b=b \sim a$ and (ii) $a \otimes(a \sim b)=\tilde{b}$.

Let $\mathcal{E}=\left(E, \otimes_{\mathcal{E}}, \wedge_{\mathcal{E}}, \sim_{\mathcal{E}}, 1_{\mathcal{E}}\right)$ and $\mathcal{G}=\left(G, \otimes_{\mathcal{G}}, \wedge_{\mathcal{G}}, \sim_{\mathcal{G}}, 1_{\mathcal{G}}\right)$ be two $E Q-$ algebras. A map $f: E \rightarrow G$ is an $E Q$-homomorphism, if for any $a, b \in E$, $f\left(a \otimes_{\mathcal{E}} b\right)=f(a) \otimes_{\mathcal{G}} f(b), f\left(a \wedge_{\mathcal{E}} b\right)=f(a) \wedge_{\mathcal{G}} f(b), f\left(a \sim_{\mathcal{E}} b\right)=f(a) \sim_{\mathcal{G}} f(b)$,

${ }^{1}$ Given an algebra $\langle E, F\rangle$, where $F$ is a set of operations on $E$ and $F^{\prime} \subseteq F$, then the algebra $\left\langle E, F^{\prime}\right\rangle$ is called the $F^{\prime}$-reduct of $\langle E, F\rangle$. 
and $f\left(1_{\mathcal{E}}\right)=1_{\mathcal{G}}$. A nonempty subset $F$ of $\mathcal{E}$ is called a filter of $\mathcal{E}$ if for any $a, b, c \in E:(\mathrm{F} 1)$ if $a \in F$ and $a \leqslant b$, then $b \in F$, (F2) if $a, b \in F$, then $a \otimes b \in F,(\mathrm{~F} 3)$ if $a \sim b \in F$, then $(a \otimes c) \sim(b \otimes c) \in F$.

Proposition 2.4 ([19]). Let $f: \mathcal{E} \rightarrow \mathcal{G}$ be an $E Q$-homomorphism. If $\mathcal{G}$ is separated, then $\operatorname{ker}(f)=\{a \in E \mid f(a)=1\}$ is a filter of $\mathcal{E}$.

Proposition 2.5 ([19]).

(i) Let $\left\{F_{i} \mid i \in I\right\}$ be a family of filters of an $E Q$-algebra $\mathcal{E}$. Then $\bigcap_{i \in I} F_{i}$ is a filter of $\mathcal{E}$.

(ii) Let $\left\{\mathcal{E}_{i} \mid i \in I\right\}$ be a family of $E Q$-algebras and $F_{i}$ be a filter of $E_{i}$ for any $i \in I$. Then $F=\prod F_{i}$ is a filter of $\mathcal{E}=\prod \mathcal{E}_{i}$.

THEOREM 2.6 ([14]). Let $F$ be a filter of EQ-algebra $\mathcal{E}$. A binary relation $\approx_{F}$ on $E$ which is defined by $a \approx_{F} b$ if and only if $a \sim b \in F$, is $a$ congruence relation on $\mathcal{E}$ and $\mathcal{E} / F=\left(E / F, \wedge_{F}, \otimes_{F}, \sim_{F}, F\right)$ is a separated $E Q$-algebra, where, for any $a, b \in E$, we have,

$$
[a] \wedge_{F}[b]=[a \wedge b], \quad[a] \otimes_{F}[b]=[a \otimes b], \quad[a] \sim_{F}[b]=[a \sim b] .
$$

A binary relation $\leqslant_{F}$ on $E / F$ which is defined by $[a] \leqslant_{F}[b]$ if and only if $[a] \wedge_{F}[b]=[a]$ is a partial order on $E / F$ and for any $[a],[b] \in \mathcal{E} / F$, $[a] \leqslant_{F}[b]$ if and only if $a \rightarrow b \in F$.

THEOREM 2.7. [19] Let $X$ be a nonempty subset of a multiplicatively relative EQ-algebra $\mathcal{E}$. Then

$$
\langle X\rangle=\left\{a \in E \mid \exists n \in \mathbb{Z}^{+}, x_{i} \in X \quad \text { s.t. } x_{1} \otimes x_{2} \otimes \cdots \otimes x_{n} \leqslant a\right\}
$$

is a generated filter by $X$.

Now, we present some definitions and results in category theory which will be used in this paper (see $[1,2,3,8,15]$ ). A category consists of objects: $A, B, X, \ldots$ and morphisms (arrows): $f, g, h, \ldots$ For each morphism $f$, there are given objects $\operatorname{dom}(f), \operatorname{cod}(f)$ called the domain and codomain of $f$. We write $f: A \rightarrow B$ to indicate that $A=\operatorname{dom}(f)$ and $B=\operatorname{cod}(f)$. Given morphisms $f: A \rightarrow B$ and $g: B \rightarrow C$, where $\operatorname{cod}(f)=\operatorname{dom}(g)$ there is given a morphism $g \circ f: A \rightarrow C$ called the composite of $f$ and $g$. For each object $A$, there is given a morphism $i d_{A}: A \rightarrow A$ called the identity morphism of $A$. These data are required to satisfy the laws, for 
all $f: A \rightarrow B, g: B \rightarrow C, h: C \rightarrow D, h \circ(g \circ f)=(h \circ g) \circ f$ and $f \circ i d_{A}=f=i d_{B} \circ f$.

A category $\mathcal{C}$ is called locally small if for all objects $X, Y$ in $\mathcal{C}$, the collection $\operatorname{Hom}_{\mathcal{C}}(X, Y)=\{f \in \mathcal{C} \mid f: X \rightarrow Y\}$ is a set (called a hom-set). A category is said to be small if its objects form a set. A category $\mathcal{C}$ is called connected, if for all objects $X, Y$ in $\mathcal{C}, \operatorname{Hom}_{\mathcal{C}}(X, Y) \neq \emptyset$. A functor $F$ from a category $\mathcal{C}$ to a category $\mathcal{D}$ is a prescription that assigns to every object $A$ of $\mathcal{C}$ an object $F(A)$ of $\mathcal{D}$, and to every morphism $\alpha: A \rightarrow B$ of $\mathcal{C}$ a morphism $F(\alpha): F(A) \rightarrow F(B)$ of $\mathcal{D}$, such that (i): $F\left(i d_{A}\right)=i d_{F(A)}$, for every object $A$ of $\mathcal{C}$ and (ii): if $\beta \circ \alpha$ is defined in $\mathcal{C}$, then $F(\beta) \circ F(\alpha)$ is defined in $\mathcal{D}$ and $F(\beta) \circ F(\alpha)=F(\beta \circ \alpha)$. A monomorphism (also called a monic morphism) is a left-cancellative morphism. That is, a morphism $f: X \rightarrow Y$ such that for all objects $Z$ and all morphisms $g_{1}, g_{2}: Z \rightarrow X$, if $f \circ g_{1}=f \circ g_{2}$, then $g_{1}=g_{2}$. An epimorphism (also called an epic morphism) is a morphism $f: X \rightarrow Y$ that is right-cancellative in the sense that, for all objects $Z$ and all morphisms $g_{1}, g_{2}: Y \rightarrow Z$, if $g_{1} \circ f=g_{2} \circ f$, then $g_{1}=g_{2}$. An initial object is an object $I$ such that for every object $X$, there exists precisely one morphism $I \rightarrow X$. A terminal object is an object $T$ such that for every object $X$, there exists precisely one morphism $X \rightarrow T$. If an object is both initial and terminal, it is called a zero object. An object $Q$ in a category $\mathcal{C}$ is said to be injective if for every monomorphism $f: X \rightarrow Y$ and every morphism $g: X \rightarrow Q$ there exists a morphism $h: Y \rightarrow Q$ such that $h \circ f=g$. If $B$ is an object of a category $\mathcal{C}$, then by a subobject of $B$ we mean a pair of $(A, f)$ consisting of an object $A$ of $\mathcal{C}$ and a morphism $f: A \rightarrow B$ that is monic. Let $X$ be a set (called a basis), $A$ be an object, and $i: X \rightarrow A$ be an injective map between sets (called the canonical insertion). We say that $A$ is the free object on $X$ (with respect to $i$ ) if and only if it satisfies the following universal property: for any object $B$ and any map between sets $f: X \rightarrow B$, there exists a unique morphism $g: A \rightarrow B$ such that $f=g \circ i$. Let $A_{1}$ and $A_{2}$ be two algebras of the same type $F$. The product $A_{1} \times A_{2}$ is an algebraic structure whose universe is the set $A_{1} \times A_{2}$, such that for $f \in F_{n}$ and $a_{i} \in A_{1}, a_{i}^{\prime} \in A_{2}, 1 \leqslant i \leqslant n$,

$$
f^{A_{1} \times A_{2}}\left(\left\langle a_{1}, a_{1}^{\prime}\right\rangle, \cdots,\left\langle a_{n}, a_{n}^{\prime}\right\rangle\right)=\left\langle f^{A_{1}}\left(a_{1}, \ldots, a_{n}\right), f^{A_{2}}\left(a_{1}^{\prime}, \ldots, a_{n}^{\prime}\right)\right\rangle .
$$

Let $A$ and $B$ be two objects and $f, g: A \rightarrow B$ be two morphisms. The equalizer of $f$ and $g$ is an object $E$ and a morphism $e q: E \rightarrow A$ satisfying $f \circ e q=g \circ e q$ such that, for any object $O$ and morphism $m: O \rightarrow A$, 
if $f \circ m=g \circ m$, then there exists a unique morphism $u: O \rightarrow E$ such that $e q \circ u=m$. Let $A$ be an object and $f: Y \rightarrow A$ and $g: Z \rightarrow A$ be two morphisms. The pullback of $f, g$ is an object $P$ with to morphisms $p_{1}: P \rightarrow Y$ and $p_{2}: P \rightarrow Z$ such that $f \circ p_{1}=g \circ p_{2}$ and for any $q_{1}: Q \rightarrow Y$ and $q_{2}: Q \rightarrow Z$ with $f \circ q_{1}=g \circ q_{2}$, there exists a unique $u: Q \rightarrow P$ such that $q_{1}=p_{1} \circ u$ and $q_{2}=p_{2} \circ u$. Let $\mathcal{J}$ and $\mathcal{C}$ be categories. A diagram of type $\mathcal{J}$ in $\mathcal{C}$ is a functor $D: \mathcal{J} \rightarrow \mathcal{C}$. We will write the objects in the index category $\mathcal{J}$ lower case, $i, j, \cdots$ and the values of the functor $D: \mathcal{J} \rightarrow \mathcal{C}$ in the form $D_{i}, D_{j}$, etc. If $\mathcal{J}$ is a small category, then $D: \mathcal{J} \rightarrow \mathcal{C}$ is a small diagram. A cone to a diagram $D$ consists of an object $C$ in $\mathcal{C}$ and a family of morphisms in $\mathcal{C}, c_{j}: C \rightarrow D_{j}$ one for each object $j \in \mathcal{J}$, such that for each morphism $\alpha: i \rightarrow j$ in $\mathcal{J}$, such that $D_{\alpha} \circ c_{i}=c_{j}$. A morphism of cones $\vartheta:\left(C, c_{j}\right) \rightarrow\left(C^{\prime}, c_{j}^{\prime}\right)$ is a morphism $\vartheta$ in $\mathcal{C}$ such that for any $j \in \mathcal{J}$, $c_{j}=c_{j}^{\prime} \circ \vartheta$. Thus, we have an apparent category $\operatorname{Cone}(D)$ of cones to $D$. A limit for a diagram $D: J \rightarrow C$ is a terminal object in Cone $(D)$. A category $\mathcal{C}$ is called small-complete if all small diagrams in $\mathcal{C}$ have limits in $\mathcal{C}$.

Suppose that $\left(A_{i}\right)_{i \in I}$ is a family of subobjects of a given object $B$. Constructing a category $\mathcal{K}$ as follows: for the objects of $\mathcal{K}$ take those subobjects $(D, d)$ of $B$ for which there exists a commutative triangle as follows.

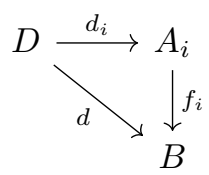

For the morphisms from $(D, d)$ to $(E, e)$ take those morphisms $\alpha: D \rightarrow E$ in $\mathcal{C}$ such that the following diagram is commutative.

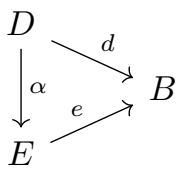

The terminal object in $\mathcal{K}$ is called an intersection of the family $\left(A_{i}, f_{i}\right)_{i} \in I$ of subobjects of $B$.

Proposition 2.8 ([3]). A category $\mathcal{C}$ has finite products if and only if it has a terminal object and every pair of objects in $\mathcal{C}$ has a product. 
TheOREM $2.9([2,3])$. Let $\mathcal{C}$ be a category. The following statements are equivalent:

(i) $\mathcal{C}$ has finite products and equalizers.

(ii) $\mathcal{C}$ has finite products and finite intersections.

(iii) $\mathcal{C}$ has pullbacks and a terminal object.

(iv) $\mathcal{C}$ has all finite limits.

Let $A$ and $B$ be two objects and $f, g: A \rightarrow B$ be two morphisms. The coequalizer of $f$ and $g$ is an object $Q$ and a morphism $q: B \rightarrow Q$ such that $q \circ f=q \circ g$. Moreover, for any object $Q^{\prime}$ and morphism $q^{\prime}: B \rightarrow Q^{\prime}$ there exists a unique morphism $u^{\prime}: Q \rightarrow Q^{\prime}$ such that $u^{\prime} \circ q=q^{\prime}$. Let $X$ be an object and $f: X \rightarrow Y$ and $g: X \rightarrow Z$ be two morphisms. The pushout of $f, g$ is an object $P$ with to morphisms $i_{1}: Y \rightarrow P$ and $i_{2}: Z \rightarrow P$ such that $i_{1} \circ f=i_{2} \circ g$ and for any $q_{1}: Y \rightarrow Q$ and $q_{2}: Z \rightarrow Q$ with $q_{1} \circ f=q_{2} \circ g$, there exists a unique $u: P \rightarrow Q$ such that $q_{1}=u \circ i_{1}$ and $q_{2}=u \circ i_{2}$.

TheOrem 2.10 ([8]). A nonempty class $K$ of algebraic structures of type $F$ is called a variety if it is closed under subalgebras, homomorphic images, and direct products.

THEOREM 2.11 ([8]). Every variety has free objects.

Notation. From now on, in this paper, $\mathcal{E}=(E, \wedge, \otimes, \sim, 1)$ or simply $\mathcal{E}$ is an $E Q$-algebra, unless otherwise state.

\section{Category of $E Q$-algebras}

If we consider $E Q$-algebras and $E Q$-homomorphisms between them as objects and morphisms, then class of all $E Q$-algebras and the $E Q$-homomorphisms with the usual composition of maps forms a locally small category which is denoted by $\mathcal{E} \mathcal{Q}$. In the rest of this article, we study the morphims, objects, limits and colimits of $\mathcal{E} \mathcal{Q}$.

\subsection{Morphisms}

In this subsection, we give the conditions that when an injective $E Q$ homomorphism is monic. Also, we show that an onto $E Q$-homomorphism is epic but the connverse is not true, in general. 
Proposition 3.1. $\mathcal{E} \mathcal{Q}$ is connected.

Proof: Let $\mathcal{E}$ and $\mathcal{G}$ be two $E Q$-algebras. Then the map $e: \mathcal{E} \rightarrow \mathcal{G}$, where for any $a \in E, e(a)=1_{\mathcal{G}}$ is a homomorphism. Thus, $\operatorname{Hom}(\mathcal{E}, \mathcal{G}) \neq \emptyset$.

Proposition 3.2. Let $f: \mathcal{E} \rightarrow \mathcal{G}$ and $\operatorname{ker} f=\left\{a \in E \mid f(a)=1_{\mathcal{G}}\right\}=\left\{1_{\mathcal{E}}\right\}$. If $\mathcal{E}$ is separated, then $f$ is injective.

Proof: Suppose that for some $a, b \in E, f(a)=f(b)$. Since $f$ is a homomorphism, by $(E 3)$ we have, $1_{\mathcal{G}}=f(a) \sim f(b)=f(a \sim b)$. Then $a \sim b \in \operatorname{ker} f$ and so $a \sim b=1$. Since $\mathcal{E}$ is separated, we have $a=b$ and $f$ is injective.

In the following example, we show that the separated condition in Proposition 3.2 is necessary.

Example 3.3. Let $H=\{0, a, b, 1\}$ be a chain where $0 \leqslant a \leqslant b \leqslant 1$. For any $x, y \in H$, we define the operations $\otimes$ and $\sim$ on $H$ as Table 1 and Table 2 :

Table 1

\begin{tabular}{l|llll}
$\otimes$ & 0 & $a$ & $b$ & 1 \\
\hline 0 & 0 & 0 & 0 & 0 \\
$a$ & 0 & 0 & 0 & $a$ \\
$b$ & 0 & 0 & 0 & $b$ \\
1 & 0 & $a$ & $b$ & 1
\end{tabular}

\section{Table 2}

\begin{tabular}{c|cccc}
$\sim$ & 0 & $a$ & $b$ & 1 \\
\hline 0 & 1 & 0 & 0 & 0 \\
$a$ & 0 & 1 & $a$ & $a$ \\
$b$ & 0 & $a$ & 1 & 1 \\
1 & 0 & $a$ & 1 & 1
\end{tabular}

Then $\mathcal{H}=(H, \wedge, \otimes, \sim, 1)$ is a non-separated $E Q$-algebra. Let $G=$ $\{0, d, 1\}$ be a chain where $0 \leqslant d \leqslant 1$. For any $x, y \in G$, we define the operations $\otimes$ and $\sim$ on $G$ as Table 3 and Table 4 :

Table 3

\begin{tabular}{c|lll}
$\otimes$ & 0 & $d$ & 1 \\
\hline 0 & 0 & 0 & 0 \\
$d$ & 0 & 0 & $d$ \\
1 & 0 & $d$ & 1
\end{tabular}

Table 4

\begin{tabular}{c|ccc}
$\sim$ & 0 & $d$ & 1 \\
\hline 0 & 1 & 0 & 0 \\
$d$ & 0 & 1 & 1 \\
1 & 0 & 1 & 1
\end{tabular}


Then $\mathcal{G}=(G, \wedge, \otimes, \sim, 1)$ is an $E Q$-algebra and $f: H \rightarrow G$ is an $E Q$ homomorphism where $f(0)=f(a)=0, f(b)=d$ and $f(1)=1$. Thus, it is clear that $\operatorname{ker} f=\{1\}$ but $f$ is not injective.

Proposition 3.4. Let $f: \mathcal{E} \rightarrow \mathcal{G}$. If $f$ is injective, then $k e r f=\left\{1_{\mathcal{E}}\right\}$.

Theorem 3.5. Let $f \in \operatorname{Hom}(\mathcal{E}, \mathcal{G})$ in $\mathcal{E} \mathcal{Q}$.

(i) If $f$ is injective, then $f$ is monic.

(ii) If $\mathcal{E}$ is a separated EQ-algebra and $f$ is monic, then $f$ is an injective map.

(iii) If $f$ is onto, then $f$ is epic.

Proof: (i) The proof is clear.

(ii) Let $H=\left\{a \in E \mid f(a)=1_{\mathcal{G}}\right\}$. It is easy to see that $\mathcal{H}=\left(H, \otimes_{\mathcal{H}}, \wedge\right.$, $\left.\sim_{\mathcal{H}}, 1_{\mathcal{E}}\right)$ is a sub-algebra of $\mathcal{E}$. Suppose $i: \mathcal{H} \rightarrow \mathcal{E}$ is an inclusion morphism and $g: \mathcal{H} \rightarrow \mathcal{E}$ is an $E Q$-homomorphism such that for any $a \in H, g(a)=$ $1_{\mathcal{E}}$. Since $f$ is monic if $f \circ i=f \circ g$, then $H=\{1\}$. Since $\mathcal{E}$ is separated, by Proposition 3.2, $f$ is injective.

(iii) Suppose that $g, h: \mathcal{G} \rightarrow \mathcal{H}$ are two morphisms such that $g \circ f=h \circ f$. Since $f$ is onto, for any $b \in G$, there is $a \in E$ where $f(a)=b$. Thus, for any $b \in G$,

$$
g(b)=g(f(a))=g \circ f(a)=h \circ f(a)=h(f(a))=h(b) .
$$

Hence $f$ is epic.

In the following example we show that the converse of Theorem 3.5 (iii) is not true, in general.

Example 3.6. Let $E=\{0, a, b, c, d, 1\}$ be a lattice with a Hesse diagram as Figure 1 . For any $x, y \in E$, we define the operations $\otimes$ and $\sim$ on $E$ as Table 5 and Table 6 :

Then $\mathcal{E}=(E, \wedge, \otimes, \sim, 1)$ is a good $E Q$-algebra. Let $\mathcal{H}$ be the $E Q$ algebra as in Example 3.3. Then the map $f: H \rightarrow E$ where $f(0)=0$, $f(a)=b, f(b)=a$ and $f(1)=1$ is a non-onto homomorphism. Let id $: E \rightarrow E$ be the identity map and $t: E \rightarrow E$ be the trivial $E Q$ homomorphism. It is clear that $h \neq g$. Since $i d \circ f(a)=b$ and $t \circ f(a)=1$, we get $h \circ f \neq g \circ f$. 


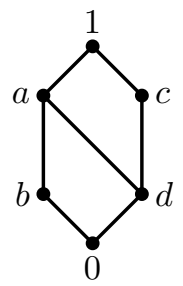

\section{Figure 1}

Table 5

\begin{tabular}{l|llllll}
$\otimes$ & 0 & $a$ & $b$ & $c$ & $d$ & 1 \\
\hline 0 & 0 & 0 & 0 & 0 & 0 & 0 \\
$a$ & 0 & $b$ & $b$ & $d$ & 0 & $a$ \\
$b$ & 0 & $b$ & $b$ & 0 & 0 & $b$ \\
$c$ & 0 & $d$ & 0 & $c$ & $d$ & $c$ \\
$d$ & 0 & 0 & 0 & $d$ & 0 & $d$ \\
1 & 0 & $a$ & $b$ & $c$ & $d$ & 1
\end{tabular}

Table 6

\begin{tabular}{c|cccccc}
$\sim$ & 0 & $a$ & $b$ & $c$ & $d$ & 1 \\
\hline 0 & 1 & $d$ & $c$ & $b$ & $a$ & 0 \\
$a$ & $d$ & 1 & $a$ & $d$ & $c$ & $a$ \\
$b$ & $c$ & $a$ & 1 & 0 & $d$ & $b$ \\
$c$ & $b$ & $d$ & 0 & 1 & $a$ & $c$ \\
$d$ & $a$ & $c$ & $d$ & $a$ & 1 & $d$ \\
1 & 0 & $a$ & $b$ & $c$ & $d$ & 1
\end{tabular}

\subsection{Objects}

In this subsection, we show that $\mathcal{E} \mathcal{Q}$ has zero objects and introduce the free $E Q$-algebra on the singleton.

TheOrem 3.7. In $\mathcal{E} \mathcal{Q},\{1\}$ is the zero object.

Proof: Let $\mathcal{E}$ be an arbitrary $E Q$-algebra. Then $f:\{1\} \rightarrow \mathcal{E}$ where $f(1)=1_{\mathcal{E}}$ is an $E Q$-homomorphism. On the other hand, let $g: \mathcal{E} \rightarrow\{1\}$ be a map where for any $a \in E, g(a)=1$. Since $g$ preserves the operations $\otimes, \wedge$ and $\sim$, we obtain that $g$ is an $E Q$-homomorphism.

Corollary 3.8. The zero objects are the only injective object in $\mathcal{E} \mathcal{Q}$.

In [14], Novák and El-zekey proved that the class of $E Q$-algebras is a variety. Thus, by Theorem 2.11, there exists a free $E Q$-algebra on a given set.

In [3], Blyth showed that the free monoid on a singleton is isomorphic to the additive monoid $\mathbb{Z}^{+}$. So we can consider free monoid on element $\{x\}$, by $\left(\mathcal{E}_{x}, \otimes\right)$ where $\mathcal{E}_{x}=\left\{e=x^{0}, x^{1}, x^{2}, \cdots, x^{i}, \cdots\right\}$. For any $i \in \mathbb{N}$, 
$x^{i} \otimes x^{j}=x^{i+j}$ and $e$ is the identity element. Now, we define a relation on $\mathcal{E}_{x}$ as follows: for any $x^{i}, x^{j} \in \mathcal{E}_{x}$, we say that $x^{i} \leqslant{ }_{f} x^{j}$ if and only if $j \leqslant i$.

Lemma 3.9. Let $\left(\mathcal{E}_{x}, \otimes\right)$ be a free monoid on $\{x\}$.

(i) The relation $\leqslant_{f}$ on $\mathcal{E}_{x}$ is an order.

(ii) For any $i, j, k \in \mathbb{Z}^{+}$, if $x^{i} \leqslant_{f} x^{j}$, then $x^{i} \otimes x^{k} \leqslant_{f} x^{j} \otimes x^{k}$.

Proof: (i) We show that $\leqslant_{f}$ is reflexive, antisymmetric, and transitive. Since $\mathbb{Z}$ is an ordered set, for any $i \in \mathbb{Z}^{+}$, we have $x^{i} \leqslant_{f} x^{i}$ and $\leqslant_{f}$ is reflexive.

Suppose that for $i, j \in \mathbb{N}, x^{i} \leqslant f x^{j}$ and $x^{j} \leqslant f x^{i}$. By definition of $\leqslant f$, we obtain $j \leqslant i$ and $i \leqslant j$. Thus $i=j$, then $x^{i}=x^{j}$ and so $\leqslant_{f}$ is antisymmetric.

Suppose for some $i, j, k \in \mathbb{N}, x^{i} \leqslant f x^{j}$ and $x^{j} \leqslant_{f} x^{k}$. By definition of $\leqslant_{f}$, we have $j \leqslant i$ and $k \leqslant j$. Hence $k \leqslant i, x^{i} \leqslant{ }_{f} x^{k}$ and so $\leqslant_{f}$ is transitive.

Since $\mathbb{N}$ is a chain, we can see that $\mathcal{E}=\left(E, \leqslant_{f}\right)$ is a chain with maximum element. Therefore, it is meet semilattice with upper bound.

(ii) Now suppose that $x^{i} \leqslant f_{f} x^{j}$, then $j \leqslant i$. For any $k \in \mathbb{Z}^{+}, j+k \leqslant i+k$ and so $x^{i+k} \leqslant x^{j+k}$. Thus, $x^{i} \otimes x^{k} \leqslant f x^{j} \otimes x^{k}$. Therefore, $\leqslant f$ is an order relation on $\mathcal{E}$.

TheOREM 3.10. Let $X=\{x\}$ be a set and $\mathcal{E}=(E, \otimes, \wedge)$ be a free monoid on $X$ with an order we define in Lemma 3.9. If for any $i, j \in \mathbb{Z}^{+}$, we define a fuzzy equality on $\mathcal{E}$ as $x^{i} \sim x^{j}=x^{|i-j|}$, then $\mathcal{E}_{x}=(E, \otimes, \wedge, \sim, e)$ is an EQ-algebra.

Proof: By Lemma 3.9, (E1) and $(E 2)$ are satisfied. For any $i \in \mathbb{Z}^{+}$, $x^{i} \sim x^{i}=x^{|i-i|}=x^{0}=e$, and so $(E 3)$ is satisfied. Without loss of generality, in the rest of proof we suppose that $i \leqslant j \leqslant k \leqslant w$. Then

$$
\left(\left(x^{i} \wedge x^{j}\right) \sim x^{k}\right) \otimes\left(x^{w} \sim x^{i}\right)=\left(x^{j} \sim x^{k}\right) \otimes\left(x^{w} \sim x^{i}\right)=x^{w+k-(i+j)} .
$$

On the other hand, $x^{k} \sim\left(x^{j} \wedge x^{w}\right)=x^{w-k}$. Moreover, since $i, j \leqslant k$, we have $i+j \leqslant 2 k$ and so $w-k \leqslant w+k-(i+j)$. Thus, $x^{w+k-(i+j)} \leqslant x^{w-k}$. Hence (E4) holds.

We can see that $\left(x^{i} \sim x^{j}\right) \otimes\left(x^{k} \sim x^{w}\right)=x^{w+j-(i+k)}$ and $\left(x^{i} \sim x^{k}\right) \sim$ $\left(x^{j} \sim x^{w}\right)=x^{|(w-j)-(k-i)|}$. To show that $(E 5)$ is satisfied, we consider two following cases. 
Case 1. If $k+j \leqslant w+i$, then $k+j-(w+i) \leqslant w+j-(i+k)$ and so

$$
\left(x^{i} \sim x^{j}\right) \otimes\left(x^{k} \sim x^{w}\right) \leqslant\left(x^{i} \sim x^{k}\right) \sim\left(x^{j} \sim x^{w}\right) .
$$

Case 2. If $w+i \leqslant k+j$, then $x^{w+j-(i+k)} \leqslant x^{(w-j)-(k-i)}$ and so (E5) holds.

Moreover, since $j-i \leqslant k-i$, we get that $x^{k-i} \leqslant x^{j-i}$ and so $\left(x^{i} \wedge x^{j} \wedge x^{k}\right) \sim$ $x^{i} \leqslant\left(x^{i} \wedge x^{j}\right) \sim x^{i}$. Thus, (E6) is satisfied.

Also, from $i, j \leqslant j+i$, we have $x^{i} \otimes x^{j} \leqslant x^{i} \wedge x^{j}$, and (E7) is satisfied. Therefore, $\mathcal{E}_{x}=(E, \otimes, \wedge, \sim, e)$ is an $E Q$-algebra.

Remark 3.11. For any $i, j \in \mathbb{Z}^{+}$, the implication operation on $\mathcal{E}_{x}$ is

$$
x^{i} \rightarrow x^{j}= \begin{cases}e & j \leqslant i \\ x^{j-i} & i<j .\end{cases}
$$

Proposition 3.12. Let $X=\{x\}$ be a set. Then the following statements hold:

(i) $\mathcal{E}_{x}$ is a good $E Q$-algebra.

(ii) $\mathcal{E}_{x}$ is a residuated $E Q$-algebra.

(iii) $\mathcal{E}_{x}$ is an $\ell E Q$-algebra.

(iv) $\mathcal{E}_{x}$ is a multiplicatively relative $E Q$-algebra.

Proof: (i) By the definition of $\sim$ on $\mathcal{E}_{x}$, the proof is clear.

(ii) Let $i, j, w \in \mathbb{Z}^{+}$. If $w \leqslant i+j$, then $\left(x^{i} \otimes x^{j}\right) \rightarrow x^{w}=e$. Thus, we consider two following cases.

Case 1. If $w \leqslant j$, then $x^{j} \rightarrow x^{w}=e$ and so for any $i \in \mathbb{Z}^{+}$, we have

$$
\left(x^{i} \otimes x^{j}\right) \rightarrow x^{w}=x^{i} \rightarrow\left(x^{j} \rightarrow x^{w}\right)=e .
$$

Case 2. If $j<w$, then $x^{j} \rightarrow x^{w}=x^{w-j}$ and since $w-j \leqslant i$ we have $x^{i} \rightarrow\left(x^{j} \rightarrow x^{w}\right)=e$.

Now, if $i+j<w$, then $\left(x^{i} \otimes x^{j}\right) \rightarrow x^{w}=x^{w-(i+j)}$. Moreover, since $i<w$ and $j<w$, we get that $x^{i} \rightarrow\left(x^{j} \rightarrow x^{w}\right)=x^{w-j-i}$. Hence, by Proposition $2.1(i i), \mathcal{E}_{x}$ is residuated. 
(iii) Without loss of generality, we consider that $i \leqslant j \leqslant k \leqslant w$. Since $2 i \leqslant w+j$, we have

$$
\left(\left(x^{i} \vee x^{j}\right) \sim x^{k}\right) \otimes\left(x^{w} \sim x^{i}\right) \leqslant\left(\left(x^{j} \vee x^{w}\right) \sim x^{k}\right) .
$$

(iv) By Lemma 3.9, we know that $\mathcal{E}_{x}$ is a chain. Then by Proposition 2.2, it is multiplicatively relative.

Remark 3.13. Since $\mathcal{E}_{x}$ dose not have least element, it is not a residuated lattice.

In the following example, we show that $\mathcal{E}_{x}$ is not free in general.

Example 3.14. Let $\mathcal{E}$ be an $E Q$-algebra as in Example 3.6. Let $X=\{x\}$ be an arbitrary set and $h: X \rightarrow E$ be a map where $h(x)=b$. If $\mathcal{E}_{x}$ is a free $E Q$-algebra, then $f: \mathcal{E}_{x} \rightarrow \mathcal{E}$ is an $E Q$-homomorphism which $f(x)=h(x)$ and so for any $i \in \mathbb{N}$, we should have $f\left(x^{i}\right)=h(x) \otimes h(x) \otimes \cdots \otimes h(x)=b$. We claim that $f$ is not an $E Q$-algebra homomorphism. Because, for $j>i$, $f\left(x^{i} \sim x^{j}\right)=f\left(x^{j-i}\right)=b$ but, $f\left(x^{i}\right) \sim f\left(x^{j}\right)=b \sim b=1 \neq b$. Thus, $f$ is not preserves the both operations $\otimes$ and $\sim$ at the same time.

Remark 3.15. Let $X=\{x\}$ be a set and $\mathcal{E}_{x}$ be the EQ-algebra as in Theorem 3.10. For any $i, j, k \in \mathbb{Z}^{+}, x^{i} \sim x^{j}=x^{i+k} \sim x^{j+k}=\left(x^{i} \otimes x^{j}\right) \sim$ $\left(x^{j} \otimes x^{k}\right)$.

Definition 3.16. An EQ-algebra $\mathcal{E}$ is multiplicatively equal if for any $a, b, c \in E$,

$$
a \sim b=(a \otimes c) \sim(b \otimes c) .
$$

Example 3.17. By Remark 3.15, $\mathcal{E}_{x}$ is multiplcatively equal.

THEOREM 3.18. The EQ-algebra $\mathcal{E}_{x}$ is a free object on the class of good multiplicatively equal EQ-algebras.

Proof: Let $\mathcal{H}=\left(H, \otimes_{H}, \wedge_{H}, \sim_{H}, e_{H}\right)$ be a multiplicatively equal $E Q$ algebra, $X=\{x\}$ be a set and $g: X \rightarrow H$ be a map. We define a map $f: \mathcal{E}_{x} \rightarrow H$ such that for any $i \in \mathbb{N}, f\left(x^{i}\right)=g(x)^{i}$ and $f\left(e_{\mathcal{E}_{x}}\right)=e_{H}$. Now, we show that $f$ is an $E Q$-homomorphism. Let $i, j \in \mathbb{N}$,

$$
f\left(x^{i} \otimes x^{j}\right)=f\left(x^{i+j}\right)=g(x)^{i+j}=g(x)^{i} \otimes_{H} g(x)^{j} .
$$

Without loss of generality, we can consider that $i \leqslant j$ and so $g(x)^{j}=$ $g(x)^{i} \otimes g(x)^{j-i}$. By $(E 7)$, we have $g(x)^{i} \wedge_{H} g(x)^{j}=g(x)^{j}$. Thus, $f\left(x^{i} \wedge x^{j}\right)=$ $f\left(x^{j}\right)=g(x)^{j}=g(x)^{i} \wedge g(x)^{j}$. 
Since $\mathcal{H}$ is good multiplicatively equal, for any $j>i \in \mathbb{N}$, we get that,

$$
g(x)^{j-i}=e_{H} \sim g(x)^{j-i}=g(x)^{i} \sim g(x)^{j}=f\left(x^{i}\right) \sim f\left(x^{j}\right) .
$$

On the other hand, $f\left(x^{i} \sim x^{j}\right)=f\left(x^{j-i}\right)=g(x)^{j-i}$ and so $f$ preserves the operation " ". Therefore, $f$ is an EQ-homomorphism.

\subsection{Limits}

In this subsection, we show $\mathcal{E} \mathcal{Q}$ has products and also all finite limits.

TheOREM 3.19. $\mathcal{E Q}$ has

(i) product,

(ii) equilizers,

(iii) pullbacks,

(iv) all finite limits,

(v) finite intersections.

Proof: (i) Since the class of $E Q$-algebras is a variety, by Theorem 2.10, it has products. Then for any $E Q$-algebras $\mathcal{E}$ and $\mathcal{G}, \mathcal{E} \times \mathcal{G}$ with pointwise operations is an $E Q$-algebra. Thus, $\mathcal{E} \times \mathcal{G}$ with projection maps $\left(p_{1}, p_{2}\right)$ is the product of $\mathcal{E}$ and $\mathcal{G}$.

(ii) Let $f, g: \mathcal{E} \rightarrow \mathcal{G}$ be two $E Q$-homomorphisms and let $H=\{a \in \mid$ $f(a)=g(a)\}$. Since $f, g$ are homomorphisms, $\mathcal{H}=\left(H, \otimes_{\mathcal{E}}, \wedge_{\mathcal{E}}, \sim_{\mathcal{E}}, 1_{\mathcal{E}}\right)$ is an $E Q$-algebra. Let $i: \mathcal{H} \rightarrow \mathcal{E}$ be the inclusion map. Then for any $a \in H$, $f \circ i(a)=f(a)=g(a)=g \circ i(a)$.

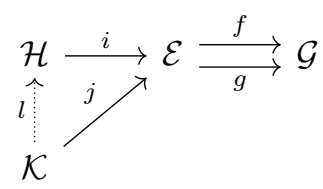

Now, suppose that $\mathcal{K}$ is an $E Q$-algebra and $j: \mathcal{K} \rightarrow \mathcal{E}$ is a morphism such that $f \circ j=g \circ j$. Then for any $x \in K, j(x) \in H$ and $\operatorname{Im}(j) \subseteq H$. Thus, we can define a morphism $l: \mathcal{K} \rightarrow \mathcal{H}$ where for any $x \in K, l(x)=j(x)$. It is clear that $l$ is an unique $E Q$-homomorphism and $i \circ l=j$. Hence $(\mathcal{H}, i)$ is the equilizer of $f, g$. 
(iii) Let $f \in \operatorname{Hom}(\mathcal{E}, \mathcal{H})$ and $g \in \operatorname{Hom}(\mathcal{G}, \mathcal{H})$. Since $\mathcal{E Q}$ has a binary product, $\mathcal{P}=\{(a, b) \in E \times G \mid f(a)=g(b)\}$ is an $E Q$-algebra and so $\left(P, p_{1}, p_{2}\right)$ is the pull back of $(f, g)$.

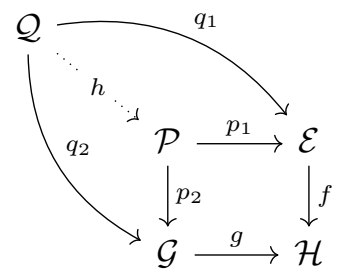

Now suppose that $\left(\mathcal{Q}, q_{1}, q_{2}\right)$ is an $E Q$-algebra with two morphisms where $f \circ q_{1}=g \circ q_{2}$. Let $h: \mathcal{Q} \rightarrow \mathcal{P}$ be a map where for any $x \in Q, h(x)=$ $\left(q_{1}(x), q_{2}(x)\right)$. Since $q_{1}$ and $q_{2}$ are homomorphisms, $h$ is homomorphism. By considering the definition of $h$, we can see that $p_{1} \circ h=q_{1}$ and $p_{2} \circ h=q_{2}$. Moreover, since $p_{1}$ and $p_{2}$ are onto, they are epic and so $h$ is unique.

(iv), (v) Since $\mathcal{E} \mathcal{Q}$ has all finite products and equlizers, by Theorem 2.9, it has all finite limits.

\subsection{Co-limits}

In this subsection, we show that $\mathcal{E} \mathcal{Q}$ does not have co-limits such as coequlizers, coproduct and pushout, in general. In the rest of this article, we introduce a method to extend any good $E Q$-algebra and by using this method we calculate coprodcuts and push out of $E Q$-algebras in special cases.

TheOREm 3.20. Let $f, g: \mathcal{E} \rightarrow \mathcal{G}$ be two EQ-homomorphisms. If $\mathcal{G}$ is a multiplicatively relative EQ-algebra, then $f, g$ have co-equilizer.

ProOF: Let

$$
\mathcal{F}=\{F \mid F \text { is a filter of } \mathcal{G} \text { such that for any } x \in E, \quad f(x) \sim g(x) \in F\} .
$$

Since $\mathcal{G}$ is a multiplicatively relative $E Q$-algebra, $\langle\operatorname{Imf} \cup \operatorname{Img}\rangle$ is a filter of $\mathcal{G}$ and so $\mathcal{F}$ is not empty. By Proposition 2.5, $\cap \mathcal{F}$ is a filter of $\mathcal{G}$. Then by Theorem 2.6, $\frac{\mathcal{G}}{\cap \mathcal{F}}$ is an $E Q$-algebra. Let $\pi: \mathcal{G} \rightarrow \mathcal{G} / \cap \mathcal{F}$ be a map such that for any $a \in G, \pi(a)=[a]$. For any $x \in E, \pi \circ f(x)=[f(x)]$ and $\pi \circ g(x)=[g(x)]$. Since $f(x) \sim g(x) \in \cap \mathcal{F}$, we have $[f(x)]=[g(x)]$. 
Suppose that there exists a separated $E Q$-algebra and a homomorphism such as $(\mathcal{J}, j)$ where $j \circ f=j \circ g$. Since, for any $x \in E, j(f(x) \sim g(x))=$ $(j \circ f(x)) \sim(j \circ g(x))=1$, we get that $f(x) \sim g(x) \in k e r j$.

Now, let $k: \mathcal{G} / \cap \mathcal{F} \rightarrow \mathcal{J}$ be a map such that $k([a])=j(a)$ for any $[a] \in \mathcal{G} / \cap \mathcal{F}$. We show that $k$ is a homomorphism.

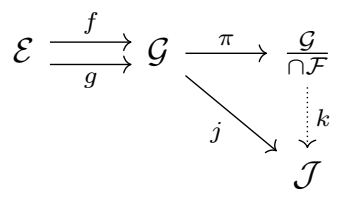

Suppose that $[a]=[b]$. By Proposition 2.6 we have, $a \sim b \in \cap \mathcal{F}$. By Proposition 2.4, kerj is a filter of $\mathcal{G}$ and so $j(a) \sim j(b)=1$. Since $\mathcal{J}$ is separated, $j(a)=j(b)$ and so $k([a])=k([b])$.

By considering the definition of $k$, it is clear that $k \circ \pi=j$ and $k$ is an unique $E Q$-homomorphism. Therefore, $(\mathcal{G} / \cap \mathcal{F}, \pi)$ is a co-equilizer.

TheOREM 3.21. Let $\mathcal{E}$ be a good. If $e \notin E$, then $\mathcal{E}^{\prime}=\left(E \cup\{e\}, \otimes_{\mathcal{E}^{\prime}}, \wedge_{\mathcal{E}^{\prime}}\right.$, $\left.\sim_{\mathcal{E}^{\prime}}, e\right)$ is a good EQ-algebra where $\otimes_{\mathcal{E}^{\prime}}, \wedge_{\mathcal{E}^{\prime}}$, and $\sim_{\mathcal{E}^{\prime}}$ define as follows:

$$
\begin{aligned}
& a \otimes_{\mathcal{E}^{\prime}} b=\left\{\begin{array}{ll}
a \otimes b & a, b \in E \\
a & a \in E, b=e \\
b & b \in E, a=e \\
e & a=b=e .
\end{array} \quad a \wedge_{\mathcal{E}^{\prime}} b= \begin{cases}a \wedge b & a, b \in E \\
a & a \in E, b=e \\
b & b \in E, a=e \\
e & a=b=e .\end{cases} \right. \\
& a \sim_{\mathcal{E}^{\prime}} b= \begin{cases}a \sim b & a, b \in E, a \neq b \\
e & a, b \in E, a=b \\
a & a \in E, b=e \\
b & b \in E, a=e \\
e & a=b=e .\end{cases}
\end{aligned}
$$

Proof: By considering the definition of $\otimes_{\mathcal{E}^{\prime}}$, we can see that $\left(\mathcal{E}^{\prime}, \otimes_{\mathcal{E}^{\prime}}, e\right)$ is a commutative monoid [4]. Also, $\left(\mathcal{E}^{\prime}, \wedge \mathcal{E}^{\prime}, e\right)$ is a meet semilattice with upper bound $e$. Now, we show $\otimes_{\mathcal{E}^{\prime}}$ is isotone with respect to $\leqslant \mathcal{E}^{\prime}$. Let $a, b \in E \cup\{e\}$ such that $a \leqslant_{\mathcal{E}^{\prime}} b$. We can consider two cases. 
Case 1. We suppose that $a \leqslant b<e$. If $c \in E$, then $a \otimes_{\mathcal{E}^{\prime}} c=a \otimes c \leqslant$ $b \otimes c=b \otimes_{\mathcal{E}^{\prime}} c$. If $c=e$, then $a \otimes_{\mathcal{E}^{\prime}} e=a \leqslant b=b \otimes_{\mathcal{E}^{\prime}} e$.

Case 2. Since for any $a \in E, a \leqslant \mathcal{E}^{\prime}$, we have $a \otimes_{\mathcal{E}^{\prime}} c=a \otimes c \leqslant c=e \otimes_{\mathcal{E}^{\prime}} c$, for any $c \in E$. If $c=e$, then $a=a \otimes_{\mathcal{E}^{\prime}} e \leqslant \mathcal{E}_{\mathcal{E}^{\prime}} e=e \otimes_{\mathcal{E}^{\prime}} e$. Thus, (E2) holds.

By considering the definition of $\sim_{\mathcal{E}^{\prime}}$, we can see that $(E 3)$ is satisfied. To show that $(E 4)$ is satisfied on $\mathcal{E}^{\prime}$ we can consider four following cases.

Case 1. Suppose that $a, b, c \in E$. Since $\mathcal{E}$ is good, we have,

$$
\begin{aligned}
\left((a \wedge b) \sim_{\mathcal{E}^{\prime}} e\right) \otimes_{\mathcal{E}^{\prime}}(c \sim a) & =(a \wedge b) \otimes(c \sim a) \\
& =((a \wedge b) \sim 1) \otimes(c \sim a) \\
& \leqslant(1 \sim(c \wedge b)) \\
& =c \wedge b \\
& =\left(e \sim \mathcal{E}^{\prime}\left(c \wedge \mathcal{E}^{\prime} b\right)\right) .
\end{aligned}
$$

Case 2. Since $\mathcal{E}$ is an $E Q$-algebra, by (E5) we have,

$$
\begin{aligned}
\left(\left(a \wedge_{\mathcal{E}^{\prime}} e\right) \sim_{\mathcal{E}^{\prime}} c\right) \otimes_{\mathcal{E}^{\prime}}\left(b \sim_{\mathcal{E}^{\prime}} a\right) & =(a \sim c) \otimes(b \sim a) \\
& \leqslant(a \sim a) \sim(c \sim b) \\
& =1 \sim(c \sim b) \\
& =\left(c \sim \mathcal{E}^{\prime}\left(b \wedge_{\mathcal{E}^{\prime}} e\right)\right) .
\end{aligned}
$$

Case 3. Since $\mathcal{E}$ is good, we have

$$
\begin{aligned}
\left(\left(a \wedge \mathcal{E}^{\prime} b\right) \sim_{\mathcal{E}^{\prime}} c\right) \otimes_{\mathcal{E}^{\prime}}\left(e \sim_{\mathcal{E}^{\prime}} a\right) & =((a \wedge b) \sim c) \otimes(1 \sim a) \\
& \leqslant(c \sim(1 \wedge b)) \\
& =\left(c \sim_{\mathcal{E}^{\prime}}\left(e \wedge_{\mathcal{E}^{\prime}} b\right)\right) .
\end{aligned}
$$

Case 4. Since $\mathcal{E}$ is good by Proposition $2.3(i i)$, we have,

$$
\begin{aligned}
\left(\left(a \wedge_{\mathcal{E}^{\prime}} e\right) \sim_{\mathcal{E}^{\prime}} e\right) \otimes_{\mathcal{E}^{\prime}}\left(d \sim_{\mathcal{E}^{\prime}} a\right) & =a \otimes(d \sim a) \leqslant d \\
& =e \sim_{\mathcal{E}^{\prime}}\left(d \wedge_{\mathcal{E}^{\prime}} e\right) .
\end{aligned}
$$

For any $a, b, c, d \in E,(E 5)$ is satisfied. Now, we show that (E6) holds. 


$$
\begin{aligned}
\left(a \sim \mathcal{E}^{\prime} b\right) \otimes_{\mathcal{E}^{\prime}}\left(c \sim_{\mathcal{E}} e\right) & =(a \sim b) \otimes(c \sim 1) \\
& \leqslant(a \sim c) \sim(b \sim 1) \\
& =\left(a \sim \mathcal{E}^{\prime} c\right) \sim_{\mathcal{E}^{\prime}}\left(b \sim_{\mathcal{E}^{\prime}} e\right) .
\end{aligned}
$$

Since for any $a, b, c \in E,\left(a \wedge_{\mathcal{E}^{\prime}} b \wedge_{\mathcal{E}^{\prime}} e\right) \sim_{\mathcal{E}^{\prime}} a=\left(a \wedge_{\mathcal{E}^{\prime}} b\right) \sim_{\mathcal{E}^{\prime}} a$ and $\left(e \wedge_{\mathcal{E}^{\prime}} b \wedge_{\mathcal{E}^{\prime}} c\right) \sim_{\mathcal{E}^{\prime}} e=\left(b \wedge_{\mathcal{E}^{\prime}} c\right) \sim_{\mathcal{E}^{\prime}} e,(E 6)$ is satisfied. For any $a \in E$, $a \otimes_{\mathcal{E}^{\prime}} e=a=a \sim_{\mathcal{E}^{\prime}} e$, and so $(E 7)$ is satisfied.

Corollary 3.22. Let $X$ be a countable chain with maximum element $x$. If $\mathcal{E}$ is a good $E Q$-algebra, then $\mathcal{E}^{\prime}=\left(E \cup X, \otimes_{\mathcal{E}^{\prime}}, \wedge_{\mathcal{E}^{\prime}}, \sim_{\mathcal{E}^{\prime}}, x\right)$ is a good $E Q$-algebra.

Proof: By induction on the cardinality of $X$ and Theorem 3.21, the proof is clear.

Theorem 3.23. Let $\mathcal{E}$ be a good EQ-algebra and $\mathcal{G}=\{e\}$. Then $\mathcal{E}$ and $\mathcal{G}$ have co-product.

Proof: By Theorem 3.21, $\mathcal{E} \cup \mathcal{G}$ is an $E Q$-algebra. Let $i_{2}: \mathcal{G} \rightarrow \mathcal{E} \cup \mathcal{G}$ be the inclusion map and $i_{1}: \mathcal{E} \rightarrow \mathcal{E} \cup \mathcal{G}$ be a map such that $i_{1}(1)=e$ and for any $a \in E-\{1\}, i_{1}(a)=a$. We claim that $\left(\mathcal{E} \cup \mathcal{G}, i_{1}, i_{2}\right)$ is the co-product of $\mathcal{E}$ and $\mathcal{G}$.

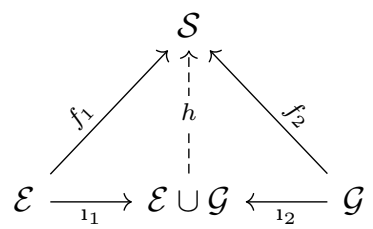

Suppose that $\left(\mathcal{S}, f_{1}, f_{2}\right)$ is an $E Q$-algebra with two homomorphisms such that $f_{1}: \mathcal{E} \rightarrow \mathcal{S}$ and $f_{2}: \mathcal{G} \rightarrow \mathcal{S}$. We define a map $h: \mathcal{E} \cup \mathcal{G} \rightarrow \mathcal{S}$ as follows:

$$
h(a)= \begin{cases}f_{1}(a) & a \in E \\ f_{2}(a) & a \in G .\end{cases}
$$

Since $f_{1}$ and $f_{2}$ are $E Q$-homomorphisms, $h$ is an $E Q$-homomorphism and by definition of $h$ we can see that $h \circ i_{1}=f_{1}$ and $h \circ i_{2}=f_{2}$ and so, $h$ is unique.

In the following example, we show that $\mathcal{E} \mathcal{Q}$ does not have co-product, in general. 
Example 3.24. Let $H=\left\{d, e_{\mathcal{H}}\right\}$ be an $E Q$-algebra and $E=\{0, a, b, 1\}$ be a chain where $0 \leqslant a \leqslant b \leqslant 1$. For any $\alpha, \beta \in E$, we define the operations $\otimes$ and $\sim$ on $E$ as Table 7 and Table 8:

Table 7

\begin{tabular}{l|llll}
$\otimes$ & 0 & $a$ & $b$ & 1 \\
\hline 0 & 0 & 0 & 0 & 0 \\
$a$ & 0 & 0 & $a$ & $a$ \\
$b$ & 0 & $a$ & 0 & $b$ \\
1 & 0 & $a$ & $b$ & 1
\end{tabular}

Table 8

\begin{tabular}{c|cccc}
$\sim$ & 0 & $a$ & $b$ & 1 \\
\hline 0 & 1 & 0 & 0 & 0 \\
$a$ & 0 & 1 & $a$ & $a$ \\
$b$ & 0 & $a$ & 1 & $b$ \\
1 & 0 & $a$ & $b$ & 1
\end{tabular}

By routine calculations, we can see that $\mathcal{E}=(E, \wedge, \otimes, \sim, 1)$ is a good $E Q$-algebra. Let $G=\{0, x, 1\}$ be a chain where $0 \leqslant x \leqslant 1$. For any $\alpha, \beta \in E$, we define the operations $\otimes$ and $\sim$ on $E$ as Table 9 and Table 10 .

Table 9

\begin{tabular}{l|lll}
$\otimes$ & 0 & $x$ & 1 \\
\hline 0 & 0 & 0 & 0 \\
$x$ & 0 & 0 & $x$ \\
1 & 0 & $x$ & 1
\end{tabular}

Table 10

\begin{tabular}{c|ccc}
$\sim$ & 0 & $x$ & 1 \\
\hline 0 & 1 & 0 & 0 \\
$x$ & 0 & 1 & $x$ \\
1 & 0 & $x$ & 1
\end{tabular}

We can see that $\mathcal{G}=(G, \wedge, \otimes, \sim, 1)$ is a good $E Q$-algebra. By routine calculations we can see that $f_{1}: E \rightarrow G$ such that $f_{1}(0)=0, f_{1}(a)=x$, and $f_{1}(b)=f_{1}(1)=1$ is an $E Q$-homomorphism. By Corollary 3.22, $\mathcal{E} \cup \mathcal{H}$ is an $E Q$-algebra. Suppose that $f_{2}: \mathcal{H} \rightarrow \mathcal{G}$ be a map where $f_{2}(d)=0$ and $f_{2}\left(e_{\mathcal{H}}\right)=1_{\mathcal{G}}$. If $\left(\mathcal{E} \cup \mathcal{H}, i_{1}, i_{2}\right)$ is the co-product of $\mathcal{E}$ and $\mathcal{H}$, there exists a homomorphism $h: \mathcal{E} \cup \mathcal{H} \rightarrow \mathcal{G}$ such that $i_{2} \circ h=f_{2}$ and $i_{1} \circ h=f_{1}$. Since $i_{2}$ is a homomorphism we get that $i_{2}\left(e_{\mathcal{H}}\right)=1_{\mathcal{G}}$. Now, we consider three cases for $i_{2}(d)$.

Case 1. Suppose that $i_{2}(d)=0$. Then we should have $h \circ i_{2}(d)=h(0)=$ $f_{2}(d)=0$. By Theorem 3.21, $a \otimes d=a$ and so we should have $h(a \otimes d)=h(a) \otimes h(d)=h(a) \otimes 0=h(a)$. According to Table 12, $h(a)=0$. On the other hand $h(0) \sim h(a)=1_{\mathcal{G}}$ and $h(0 \sim a)=$ $h(0)=0$, which means that $h$ is not a homomorphism.

Case 2. If $i_{2}(d) \in\{a, b, d, 1\}$, then $h(0)=h(a)=0$ with similar way in Case 1 , we can see that $h$ is not homomorphism. 
Case 3. Suppose that $i_{2}(d)=e_{\mathcal{H}}$. Then $h \circ i_{2}(d)=h\left(e_{\mathcal{H}}\right)=f_{2}(d)=0$. But if $h$ is a homomorphism, then we should have $h\left(e_{\mathcal{H}}\right)=1_{\mathcal{G}}$.

TheOREm 3.25. Let $\mathcal{G}$ be a good EQ-algebra and $\mathcal{H}=\{e\}$. If $f: \mathcal{E} \rightarrow \mathcal{G}$ and $g: \mathcal{E} \rightarrow \mathcal{H}$ are an arbitrary and trivial EQ-homomorphisms, respectively, then $(\mathcal{E}, f, g)$ has pushout.

Proof: By Theorem 3.23, $(\mathcal{G} \cup \mathcal{H})$ is the co-product of $\mathcal{G}$ and $\mathcal{H}$. Let $t: \mathcal{G} \rightarrow \mathcal{G} \cup \mathcal{H}$ be the trivial homomorphism. For any $a \in E$, we have $t \circ$ $f(a)=t(f(a))=e_{\mathcal{H}}=i d \circ g(a)$ and the following diagram is commutative.

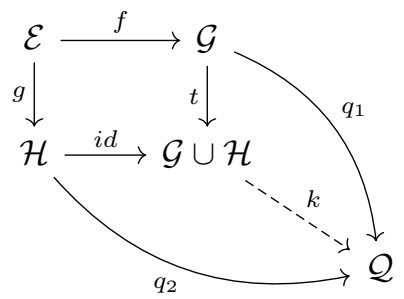

Suppose that $\left(\mathcal{Q}, q_{1}, q_{2}\right)$ is an $E Q$-algebra with two homomorphisms where $q_{1} \circ f=q_{2} \circ g$. We can see that $q_{1}$ is trivial $E Q$-homomorphism, too. If $k: \mathcal{G} \cup \mathcal{H} \rightarrow \mathcal{Q}$ is the trivial map, then the above diagram is commutative and also $k$ is unique.

\section{Conclusions and future works}

In this paper, the category of $E Q$-algebras is studied and showed that it is complete, but it is not cocomplete, in general. It is proved that the multiplicatively relative $E Q$-algebras have coequlizers and coprodut and pushout in a special case. Also, the free $E Q$-algebra on a singleton is constructed. Since every good $E Q$-algebra is an equality algebra [20], most results of this paper hold for equality algebras, too. For the future work, we can find free $E Q$-algebra on any set. Maybe, there exist some special kind of $E Q$-algebras which have co-product and pushout in general. 
Acknowledgements. The authors are very indebted to the editor and anonymous referees for their careful reading and valuable suggestions which helped to improve the readability of the paper.

This research is supported by a grant of National Natural Science Foundation of China (11971384).

\section{References}

[1] J. Adámek, H. Herrlich, G. E. Strecker, Abstract and concrete categorise, the joy of cats, no. 17 in A Wiely series OF Texts, Monographs and Tracts Pure and applied mathematics, John Wiley and Sons, New York (1990).

[2] S. Awodey, Category theory, Oxford Logic Guides, Clarendon press, Oxford (2006).

[3] T. S. Blyth, Categories, Longman, London (1986).

[4] R. A. Borzooei, M. Bakhshi, O. Zahiri, Filter theory on hyper residuated lattices, Quasigroups and Related Systems, vol. 22 (2014), pp. 33-50, DOI: https://doi.org/10.1090/S0002-9904-1935-06166-X.

[5] R. A. Borzooei, A. A. Estaji, M. Mobini, On the category of rough sets, Soft Computing, vol. 21 (2017), pp. 2201-2214, DOI: https://doi.org/10.1007/ s00500-016-2135-9.

[6] R. A. Borzooei, S. Kaviani, M. M. Zahed, Category of MV-algebras, Italian Journal of Pure and Applied Mathematics, vol. 19 (2016), pp. 61-74, DOI: https://doi.org/10.2307/2267577.

[7] R. A. Borzooei, M. Mobini, M. M. Ebrahimi, The category of soft sets, Journal of Intelligent and Fuzzy Systems, vol. 28(1) (2015), pp. 157171, DOI: https://doi.org/110.3233/IFS-141286.

[8] S. Burris, H. P. Sankappanavar, A course in universal algebra (Graduate Texts in Mathematics), no. 78 in OXFORD LOGIC GUIDES, Springer-Verlag, New York-Berlin (1981), DOI: https://doi.org/10.2307/ 2322184 .

[9] R. Cignoli, D. Mundici, An elementary presentation of the equivalence between $M V$-algebras and $\ell$-groups with strong units, Studia Logica, special issue on Many-valued logics, vol. 61 (1998), pp. 49-64. 
[10] A. DiNola, L. Leustean, Compact representations of BL-algebras, Archive for Mathematical Logic, vol. 42(8) (2003), pp. 737-761, DOI: https:// doi.org/10.1007/s00153-003-0178-y.

[11] A. Dvurečenskij, Commutative BCK-algebras with product, Demonstratio Mathematica,, vol. XXXIII(1) (2000), pp. 1-19, DOI: https://doi.org/10. $2307 / 2267577$.

[12] G. Dymek, On the category of pseudo-BCI-algebras, Demonstatio Mathematica, vol. XLVI(4) (2013), pp. 631-644, DOI: https://doi.org/10.1515/ dema-2013-0479.

[13] M. El-Zekey, Representable good EQ-algebras, Soft Computing, vol. 14(9) (2010), pp. 1011-1023, DOI: https://doi.org/10.1016/j.fss.2011.05.011.

[14] M. El-Zekey, V. Novák, R. Mesiar, On good EQ-algebras, Fuzzy Sets and Systems, vol. 178(1) (2011), pp. 1-23, DOI: https://doi.org/10.1016/j.fss. 2011.05.011.

[15] S. Maclane, Categories for the working mathematician (second ed.), vol. 5 of Graduate Texts in Mathematics, Springer-Verlag (1978), DOI: https://doi.org/10.1007/978-1-4757-4721-8.

[16] V. Novák, EQ-algebras: Primary concepts and properties, [in:] Proceedings of the Czech-Japan Seminar, Ninth Meeting, Graduate School of Information, Waseda University, Kitakyushu and Nagasaki (2006).

[17] V. Novák, EQ-algebras-based fuzzy type theory and its extensions, Logic Journal of the IGPL, vol. 19(3) (2011), pp. 512-542, DOI: https://doi. org/10.1093/jigpal/jzp087.

[18] V. Novák, B. D. Baets, EQ-algebras, Fuzzy Sets and Systems, vol. 160(20) (2009), pp. 2956-2978, DOI: https://doi.org/0.1016/j.fss.2009. 04.010.

[19] J. Yang, X. Zhang, Finite direct product of EQ-algebras, Soft Computing, vol. 23 (2019), pp. 7495-7504, DOI: https://doi.org/10.1007/s00500-01803687-5.

[20] F. Zebardast, R. A. Borzooei, M. A. Kologhani, Results on equality algebras, Information Sciences, vol. 381(1) (2017), pp. 270-282, DOI: https://doi. org/10.1016/j.ins.2016.11.027. 
On the Category of EQ-algebras

Narges Akhlaghinia

Shahid Beheshti University

Department of Mathematics

Tehran

Iran

e-mail:n_akhlaghinia@sbu.ac.ir

Mona Aaly Kologani

Shahid Beheshti University

Department of Mathematics

Tehran

Iran

e-mail: mona4011@gmail.com

\section{Rajab Ali Borzooei}

Shahid Beheshti University

Department of Mathematics

Tehran

Iran

e-mail: borzooei@sbu.ac.ir

\section{Xiao Long Xin}

Northwest University

School of Mathematics

Xi'an, 710127

People's Republic China

e-mail: xlxin@nwu.edu.cn 